\title{
OPTIMALITY AND PARAMETRIC DUALITY FOR NONSMOOTH MINIMAX FRACTIONAL PROGRAMMING PROBLEMS INVOLVING L-INVEX-INFINE FUNCTIONS
}

\author{
Anurag Jayswal*, Krishna Kummari and Vivek Singh
}

Received October 15, 2015 / Accepted July 6, 2016

\begin{abstract}
The Karush-Kuhn-Tucker type necessary optimality conditions are given for the nonsmooth minimax fractional programming problem with inequality and equality constraints. Subsequently, based on the idea of $L$-invex-infine functions defined in terms of the limiting/Mordukhovich subdifferential of locally Lipschitz functions, we obtain sufficient optimality conditions for the considered nonsmooth minimax fractional programming problem and also we provide an example to justify the existence of sufficient optimality conditions. Furthermore, we propose a parametric type dual problem and explore duality results.
\end{abstract}

Keywords: Limiting subdifferential, L-invex-infine function, minimax programming, optimality conditions, duality.

\section{INTRODUCTION}

The importance of minimax problems is well known in optimization theory as they occur in enormous numbers of applications in economics and engineers. Over the last decade much research has been conducted on sufficiency and duality for minimax fractional programming problems, which are not necessarily smooth. The interested reader is referred to $[1,2,3,4,11,13,16$, $23,24,27]$ for more information of sufficiency and duality for minimax fractional programming problems and to $[5,9,10,12,17]$ for some of its applications in practice.

There exists a generalization of convexity to locally Lipschitz functions, with derivative replaced by the Clarke generalized gradient (see e.g. [3, 4, 15, 16, 27]). Antczak and Stasiak [4] introduced a new class of nonconvex nondifferentiable functions, called locally Lipschitz $(\Phi, \rho)$ invex functions as a generalization of $(\Phi, \rho)$-invexity notion introduced by Caristi et al. [6], with the tool Clarke generalized subgradient. Later, Antczak [3] established parametric and nonparametric optimality conditions and several duality results in the sense of Mond-Weir and Wolfe for

*Corresponding author.

Department of Applied Mathematics, Indian School of Mines, Dhanbad-826 004, Jharkhand, India. E-mails: anurag_jais123@yahoo.com; krishna.maths@gmail.com; viveksingh.25jun@gmail.com 
a new class of nonconvex nonsmooth minimax programming problems involving nondifferentiable $(\Phi, \rho)$-invex functions. However, the results cannot be applied to generalized fractional programming involving equality constraints.

During the last two decades there has been an extremely rapid development in subdifferential calculus of nonsmooth analysis and which is well recognized for its many applications to optimization theory. The Mordukhovich subdifferential is a highly important notion in nonsmooth analysis and closely related to optimality conditions of locally Lipschitzian functions of optimization theory (see $[19,22]$ ). The Mordukhovich subdifferential is a closed subset of the Clarke subdifferential and this subdifferentials are in general nonconvex sets, unlike the well-known Clarke subdifferentials. Therefore, from the point of view of optimization and its applications, the descriptions of the optimality conditions and calculus rules in terms of Mordukhovich subdifferentials provide sharp results than those given in terms of the Clarke generalized gradient (see e.g. [7, 8, 18]).

Sach et al. [21] observed that the usual notion of invexity is suitable for optimization problem with inequality constraints, but it is not suitable for optimization problem with equality constraints. Therefore, Sach et al. [21] defined the notion of infine nonsmooth functions for locally Lipschitz functions, which is a generalization of invexity [14] and studied several characterizations of infineness property. Very recently, Chuong [7] introduced the concept of $L$-invex-infine functions by employing the limiting/Mordukhovich subdifferential instead of the Clarke subdifferential one which has been used before in the definitions of invex-infine functions [20, 21].

Consequently, in the present paper, we concentrate on studying nonsmooth minimax fractional programming problem with inequality and equality constraints to derive optimality conditions and duality results by means of employing $L$-invex-infine functions. Although many efforts have been made on this topic, it still remains a very attractive and challenging area of research. There are several approaches developed in the literature, see $[1,2,3,4,8,15,16,20,21,27]$ and the references therein.

The summary of the paper is as follows. Section 2 contains basic definitions and a few basic auxiliary results, which will be needed later in the sequel. Section 3 is devoted to the optimality conditions, and in Section 4 we turn to an investigation of the notion of duality for the nonsmooth minimax fractional programming problem. Here we propose a parametric type dual problem and prove weak, strong and strict converse duality theorems. The final Section 5 contains the concluding remarks and further developments.

\section{PRELIMINARIES}

In this section, we gather for convenience of reference, a number of basic definitions which will be used often throughout the sequel, and recall some auxiliary results.

Let $R^{n}$ be the $n$-dimensional Euclidean space and $R_{+}^{n}$ be its non-negative orthant. Unless otherwise stated, all the spaces in this paper are Banach whose norms are always denoted by $\|$.$\| . Given$ a space $X$, it's dual is denoted by $X^{*}$ and the canonical pairing between $X$ and $X^{*}$ is denoted by 
$\langle.,$.$\rangle . The polar cone of a set S \subset X$ is defined by $S^{\circ}=\left\{u^{*} \in X^{*}:\left\langle u^{*}, u\right\rangle \leq 0, \forall u \in S\right\}$ and the notation $\operatorname{cl} S$ represents the closure of $S$.

Definition 2.1 (Mordukhovich [18]). Given a multifunction $F: X \rightrightarrows X^{*}$ between a Banach space and its dual, the notation

$$
\begin{aligned}
\operatorname{Limsup}_{u \rightarrow \bar{u}} F(u)= & \left\{u^{*} \in X^{*}: \exists \text { sequences } u_{n} \rightarrow \bar{u} \text { and } u_{n}^{*} \stackrel{w^{*}}{\rightarrow} u^{*}\right. \\
& \text { with } \left.u_{n}^{*} \in F\left(u_{n}\right) \text { for all } n \in \mathbb{N}\right\}
\end{aligned}
$$

signifies the sequential Painlevé-Kuratowski upper/outer limit with respect to the norm topology of $X$ and the weak $k^{*}$ topology of $X^{*}$, where the notation $\stackrel{w^{*}}{\rightarrow}$ indicates the convergence in the weak ${ }^{*}$ topology of $X^{*}$ and $\mathbb{N}$ denotes the set of all natural numbers.

Definition 2.2 (Mordukhovich [18]). Given $S$ and $\epsilon \geq 0$, define the set of $\epsilon$-normals to $S$ at $\bar{u} \in S$ by

$$
\widehat{N}_{\epsilon}(\bar{u}, S)=\left\{u^{*} \in X^{*}: \underset{\substack{S \\ u}}{\operatorname{Lim} \sup } \frac{\left\langle u^{*}, u-\bar{u}\right\rangle}{\|u-\bar{u}\|} \leq \epsilon\right\},
$$

where $u \stackrel{S}{\rightarrow} \bar{u}$ means that $u \rightarrow \bar{u}$ with $u \in S$. When $\epsilon=0$, the set $\widehat{N}(\bar{u}, S)=\widehat{N}_{0}(\bar{u}, S)$ in $(1)$ is a cone called the Fréchet normal cone to $S$ at $\bar{u}$. If $\bar{u} \notin S$, we put $\widehat{N}_{\epsilon}(\bar{u}, S)=\emptyset$ for all $\epsilon \geq 0$.

Definition 2.3 (Mordukhovich [18]). The limiting/Mordukhovich normal cone to $S$ at $\bar{u} \in S$, denoted by $N(\bar{u}, S)$, is obtained from $\widehat{N}_{\epsilon}(u, S)$ by taking the sequential Painlevé-Kuratowski upper limits as

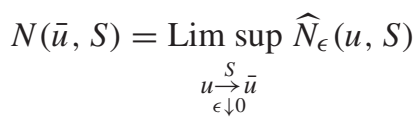

If $\bar{u} \notin S$, we put $N(\bar{u}, S)=\emptyset$. Note that one can put $\epsilon=0$ in (2) when $S$ is (locally) closed around $\bar{u}$, i.e., there is a neighborhood $U$ of $\bar{u}$ such that $S \cap \mathrm{cl} U$ is closed (see Mordukhovich [18], Theorem 1.6).

Definition 2.4 (Mordukhovich [18]). The limiting/Mordukhovich subdifferential of an extended real-valued function $\psi: X \rightarrow \bar{R}=[-\infty, \infty]$, at $\bar{u} \in X$ with $|\psi(\bar{u})|<\infty$ is defined by

$$
\partial \psi(\bar{u})=\left\{u^{*} \in X^{*}:\left(u^{*},-1\right) \in N((\bar{u}, \psi(\bar{u})), \text { epi } \psi)\right\},
$$

where epi $\psi=\{(u, \mu) \in X \times R: \mu \geq \psi(u)\}$.

If $|\psi(\bar{u})|=\infty$, one puts $\partial \psi(\bar{u})=\emptyset$. It is known (cf. Mordukhovich [18]) that when $\psi$ is a convex function, the above-defined subdifferential coincides with the subdifferential in the sense of convex analysis (cf. Rockafellar [25]).

Definition 2.5 (Mordukhovich [18]). A set $S \subset X$ is sequentially normally compact (SNC) at $\bar{u} \in S$ if for any sequence $\left(\epsilon_{n}, u_{n}, u_{n}^{*}\right) \in[0, \infty) \times S \times X^{*}$ satisfying $\epsilon_{n} \downarrow 0, u_{n} \stackrel{S}{\rightarrow} \bar{u}$ and $u_{n}^{*} \stackrel{w^{*}}{\rightarrow} 0$ with $u_{n}^{*} \in \hat{N}_{\epsilon_{n}}\left(u_{n}, S\right)$, one has $\left\|u_{n}^{*}\right\| \rightarrow 0$ as $n \rightarrow \infty$. 
In the above definition, $\epsilon_{n}$ can be omitted when $S$ is closed around $\bar{u}$. Obviously, this property is automatically satisfied in finite dimensional spaces. The reader is referred to Mordukhovich [18] for various sufficient conditions ensuring the fulfillment of the SNC property.

In the sequel of the paper, assume that $S$ is a nonempty locally closed subset of $X$, and let $I=\{1,2, \ldots, p\}, J=\{1,2, \ldots, q\}$ and $K=\{1,2, \ldots, r\}$ be index sets. In what follows, $S$ is always assumed to be $\mathrm{SNC}$ at the point under consideration.

The problem to be considered in the present analysis is the minimax fractional programming problem of the form:

$$
\begin{aligned}
& \min _{x \in \mathbb{F}} \max _{1 \leq i \leq p} \frac{f_{i}(x)}{g_{i}(x)} \\
& \text { subject to } \\
& h_{j}(x) \leq 0, \quad j \in J, \quad \ell_{k}(x)=0, \quad k \in K,
\end{aligned}
$$

where the functions $f_{i}, g_{i}, i \in I, h_{j}, j \in J$ and $\ell_{k}, k \in K$ are locally Lipschitz on $X$. The region where the constraints are satisfied (feasibility region) is given by $\mathbb{F}=\{x \in S$ : $\left.h_{j}(x) \leq 0, j \in J, \ell_{k}=0, k \in K\right\}$. Hereafter, we use the notation $f=\left(f_{1}, f_{2}, \ldots, f_{p}\right)$, $g=\left(g_{1}, g_{2}, \ldots, g_{p}\right), h=\left(h_{1}, h_{2}, \ldots, h_{q}\right)$ and $\ell=\left(\ell_{1}, \ell_{2}, \ldots, \ell_{r}\right)$.

Definition 2.6. Let $\phi(x)=\max _{i \in I} \frac{f_{i}(x)}{g_{i}(x)}, x \in X$. A point $\bar{u} \in \mathbb{F}$ is termed to be a local optimal solution of problem $(\mathrm{P})$ if there is a neighborhood $U$ of $\bar{u}$ such that

$$
\phi(\bar{u}) \leq \phi(x), \forall x \in U \cap \mathbb{F} .
$$

If the inequality in (3) holds for every $u \in \mathbb{F}$, then $\bar{u}$ is said to be a global optimal solution (or simply, optimal solution) of problem $(\mathrm{P})$.

For $\bar{u} \in S$ we put

$$
J(\bar{u})=\left\{j \in J: h_{j}(\bar{u})=0\right\}, \quad K(\bar{u})=\left\{k \in K: \ell_{k}(\bar{u})=0\right\} .
$$

Definition 2.7. The problem (P) is said to satisfy the Constraint qualification (CQ) at $\bar{u} \in S$ if there do not exist $\beta_{j} \geq 0, j \in J(\bar{u})$ and $\gamma_{k} \geq 0, k \in K(\bar{u})$, such that $\sum_{j \in J(\bar{u})} \beta_{j}+\sum_{k \in K(\bar{u})} \gamma_{k} \neq 0$ and

$$
0 \in \sum_{j \in J(\bar{u})} \beta_{j} \partial h_{j}(\bar{u})+\sum_{k \in K(\bar{u})} \gamma_{k}\left(\partial \ell_{k}(\bar{u}) \cup \partial\left(-\ell_{k}\right)(\bar{u})\right)+N(\bar{u}, S) .
$$

Remark 2.1. If we consider $\bar{u} \in \mathbb{F}, S=X$ and all the functions are continuously differentiable, then the above-defined (CQ) reduces to Mangasarian-Fromovitz constraint qualification; see e.g., Mordukhovich [18] for more details. 
Now, we define the concept of generalized convexity-affineness type for locally Lipschitz functions as follows on the lines of Chuong [7].

Definition 2.8. We say that $(f,-g, h ; \ell)$ is $L$-(strictly) invex-infine on $S$ at $\bar{u} \in S$ if for any $x \in S, x_{i}^{*} \in \partial f_{i}(\bar{u}), y_{i}^{*} \in \partial\left(-g_{i}\right)(\bar{u}), i \in I, z_{j}^{*} \in \partial h_{j}(\bar{u}), j \in J$ and $\xi_{k}^{*} \in \partial \ell_{k}(\bar{u}) \cup \partial\left(-\ell_{k}\right)(\bar{u})$, $k \in K$ there exists $v \in N(\bar{u}, s)^{\circ}$ such that

$$
\begin{gathered}
f_{i}(x)-f_{i}(\bar{u})(>) \geq\left\langle x_{i}^{*}, v\right\rangle, i \in I, \quad(x \neq \bar{u}), \\
-g_{i}(x)+g_{i}(\bar{u}) \geq\left\langle y_{i}^{*}, v\right\rangle, \quad i \in I, \\
h_{j}(x)-h_{j}(\bar{u}) \geq\left\langle z_{j}^{*}, v\right\rangle, j \in J, \\
\ell_{k}(x)-\ell_{k}(\bar{u})=w_{k}\left\langle\xi_{k}^{*}, v\right\rangle, k \in K,
\end{gathered}
$$

where $w_{k}=1$ (respectively, $w_{k}=-1$ ) whenever $\xi_{k}^{*} \in \partial \ell_{k}(\bar{u})$ (respectively, $\xi_{k}^{*} \in \partial\left(-\ell_{k}\right)(\bar{u})$ ).

In the subsequent part of this paper, we assume that $w_{k}=1$ (respectively, $w_{k}=-1$ ) whenever $\xi_{k}^{*} \in \partial \ell_{k}(\bar{u})$ (respectively, $\left.\xi_{k}^{*} \in \partial\left(-\ell_{k}\right)(\bar{u})\right)$ and $\bar{u} \in S$.

It is well known that the problem (P) is equivalent (see [27]) to the following nonfractional parametric problem:

$$
\left(\mathrm{P}_{v}\right) \quad V(v)=\min _{x \in \mathbb{F}} \max _{1 \leq i \leq p}\left\{f_{i}(x)-v g_{i}(x)\right\},
$$

where $v \in R_{+}$is a parameter.

Lemma 2.1 (Zalmai [26]). Problem (P) has an optimal solution at $\bar{u}$ with the optimal value $\bar{v}$ if and only if $V(\bar{v})=0$ and $\bar{u}$ is an optimal solution of $\left(\mathrm{P}_{\bar{v}}\right)$.

Lemma 2.2 (Zalmai [26]). For each $x \in \mathbb{F}$, one has

$$
\phi(x)=\max _{1 \leq i \leq p} \frac{f_{i}(x)}{g_{i}(x)}=\max _{\alpha \in \Lambda} \frac{\sum_{i \in I} \alpha_{i} f_{i}(x)}{\sum_{i \in I} \alpha_{i} g_{i}(x)},
$$

where $\Lambda=\left\{\alpha \in R_{+}^{p}: \sum_{i \in I} \alpha_{i}=1\right\}$.

\section{OPTIMALITY CONDITIONS}

In this section, we first derive Karush-Kuhn-Tucker type necessary conditions for (local) optimal solutions of problem (P) and then using the notion of generalized convexity-affineness-type for locally Lipschitz functions, we also establish sufficient optimality conditions.

Theorem 3.1 (Karush-Kuhn-Tucker Type Necessary Conditions). If $\tilde{x}$ is a local optimal solution of problem (P), and the constraints qualification (CQ) is satisfied at $\tilde{x}$, then there exist $\tilde{v}=\phi(\tilde{x}) \in R_{+}, \bar{\alpha} \in R_{+}^{p} \backslash\{0\}, \bar{\beta} \in R_{+}^{q}$, and $\bar{\gamma} \in R_{+}^{r}$ such that

$$
0 \in \sum_{i \in I} \bar{\alpha}_{i}\left[\partial f_{i}(\tilde{x})-\tilde{v} \partial g_{i}(\tilde{x})\right]+\sum_{j \in J} \bar{\beta}_{j} \partial h_{j}(\tilde{x})+\sum_{k \in K} \bar{\gamma}_{k}\left(\partial \ell_{k}(\tilde{x}) \cup \partial\left(-\ell_{k}\right)(\tilde{x})\right)+N(\tilde{x}, S) \text {, }
$$




$$
\begin{gathered}
\bar{\alpha}_{i}\left(f_{i}(\tilde{x})-\tilde{v} g_{i}(\tilde{x})\right)=0, i \in I, \\
\bar{\beta}_{j} h_{j}(\tilde{x})=0, j \in J .
\end{gathered}
$$

Proof. If $\tilde{x}$ is a local optimal solution of problem (P), by Lemma 2.1, it is a local optimal solution of $\left(\mathrm{P}_{\tilde{v}}\right)$ with optimal value $\tilde{v}=\max _{1 \leq i \leq p} \frac{f_{i}(\tilde{x})}{g_{i}(\tilde{x})}=\phi(\tilde{x})$. By Theorem 3.3 [8], there exist $\bar{\alpha} \in R_{+}^{p} \backslash\{0\}, \bar{\beta} \in R_{+}^{q}$, and $\bar{\gamma} \in R_{+}^{r}$ such that the conditions (4)-(6) are satisfied.

Theorem 3.2 (Sufficient Optimality Conditions). Let $(\tilde{x}, \bar{\alpha}, \bar{\beta}, \bar{\gamma}) \in \mathbb{F} \times R_{+}^{p} \backslash\{0\} \times R_{+}^{q} \times R_{+}^{r}$ satisfy the relations (4)-(6) and $\phi(\tilde{x})=\tilde{v} \in R_{+}$. Assume also that $(f,-g, h ; \ell)$ is L-invex-infine on $S$ at $\tilde{x}$. Then $\tilde{x}$ is a global optimal solution of problem $(\mathrm{P})$.

Proof. Since $(\tilde{x}, \bar{\alpha}, \bar{\beta}, \bar{\gamma}) \in \mathbb{F} \times R_{+}^{p} \backslash\{0\} \times R_{+}^{q} \times R_{+}^{r}$ satisfy the relations (4)-(6), there exist $x_{i}^{*} \in \partial f_{i}(\bar{x}), y_{i}^{*} \in \partial\left(-g_{i}\right)(\bar{x}), i \in I, z_{j}^{*} \in \partial h_{j}(\bar{x}), j \in J$ and $\xi_{k}^{*} \in \partial \ell_{k}(\bar{x}) \cup \partial\left(-\ell_{k}\right)(\bar{x}), k \in K$ such that

$$
\begin{gathered}
-\left(\sum_{i \in I} \bar{\alpha}_{i}\left[x_{i}^{*}+\tilde{v} y_{i}^{*}\right]+\sum_{j \in J} \bar{\beta}_{j} z_{j}^{*}+\sum_{k \in K} \bar{\gamma}_{k} \xi_{k}^{*}\right) \in N(\tilde{x}, S), \\
\bar{\alpha}_{i}\left(f_{i}(\tilde{x})-\tilde{v} g_{i}(\tilde{x})\right)=0, i \in I, \\
\bar{\beta}_{j} h_{j}(\tilde{x})=0, j \in J .
\end{gathered}
$$

Suppose to the contrary that $\tilde{x}$ is not a global optimal solution of $(\mathrm{P})$. Then, there exists a feasible solution $x_{0} \in \mathbb{F}$ such that

$$
\phi(\tilde{x})>\phi\left(x_{0}\right) .
$$

Using this inequality along with Lemma 2.2 and $\phi(\tilde{x})=\tilde{v}$, we get

$$
\begin{aligned}
\tilde{v} & =\phi(\tilde{x})>\phi\left(x_{0}\right) \\
& =\max _{1 \leq i \leq p} \frac{f_{i}\left(x_{0}\right)}{g_{i}\left(x_{0}\right)} \\
& =\max _{\hat{\alpha} \in \Lambda} \frac{\sum_{i \in I} \hat{\alpha}_{i} f_{i}\left(x_{0}\right)}{\sum_{i \in I} \hat{\alpha}_{i} g_{i}\left(x_{0}\right)} \\
& \geq \frac{\sum_{i \in I} \bar{\alpha}_{i} f_{i}\left(x_{0}\right)}{\sum_{i \in I} \bar{\alpha}_{i} g_{i}\left(x_{0}\right)} .
\end{aligned}
$$

Consequently, relations (8) and (10) yield

$$
\sum_{i \in I} \bar{\alpha}_{i}\left(f_{i}\left(x_{0}\right)-\tilde{v} g_{i}\left(x_{0}\right)\right)<0=\sum_{i \in I} \bar{\alpha}_{i}\left(f_{i}(\tilde{x})-\tilde{v} g_{i}(\tilde{x})\right) .
$$


By assumption, $(f,-g, h ; \ell)$ is $L$-invex-infine on $S$ at $\tilde{x}$. Then, by Definition 2.8 , there exists $v \in N(\tilde{x}, s)^{\circ}$ such that the following inequalities

$$
\begin{gathered}
f_{i}\left(x_{0}\right)-f_{i}(\tilde{x}) \geq\left\langle x_{i}^{*}, v\right\rangle, i \in I, \\
-g_{i}\left(x_{0}\right)+g_{i}(\tilde{x}) \geq\left\langle y_{i}^{*}, v\right\rangle, i \in I, \\
h_{j}\left(x_{0}\right)-h_{j}(\tilde{x}) \geq\left\langle z_{j}^{*}, v\right\rangle, j \in J, \\
\ell_{k}\left(x_{0}\right)-\ell_{k}(\tilde{x})=w_{k}\left\langle\xi_{k}^{*}, v\right\rangle, k \in K,
\end{gathered}
$$

hold for any $x_{0} \in \mathbb{F}, x_{i}^{*} \in \partial f_{i}(\tilde{x}), y_{i}^{*} \in \partial\left(-g_{i}\right)(\tilde{x}), i \in I, z_{j}^{*} \in \partial h_{j}(\tilde{x}), j \in J$ and $\xi_{k}^{*} \in \partial \ell_{k}(\tilde{x}) \cup \partial\left(-\ell_{k}\right)(\tilde{x}), k \in K$.

Since $\tilde{v} \in R_{+}$, then inequalities (12) and (13) together yield

$$
\left[f_{i}\left(x_{0}\right)-\tilde{v} g_{i}\left(x_{0}\right)\right]-\left[f_{i}(\tilde{x})-\tilde{v} g_{i}(\tilde{x})\right] \geq\left\langle\left[x_{i}^{*}+\tilde{v} y_{i}^{*}\right], v\right\rangle, i \in I .
$$

Multiplying each inequality (16) by $\bar{\alpha}_{i}, i \in I$, each inequality (14) by $\bar{\beta}_{j}, j \in J$ and each inequality (15) by $\bar{\gamma}_{k}, k \in K$, then summing resultant inequalities, we get

$$
\begin{aligned}
& \sum_{i \in I} \bar{\alpha}_{i}\left[f_{i}\left(x_{0}\right)-\tilde{v} g_{i}\left(x_{0}\right)\right]-\sum_{i \in I} \bar{\alpha}_{i}\left[f_{i}(\tilde{x})-\tilde{v} g_{i}(\tilde{x})\right] \\
+ & \sum_{j \in J} \bar{\beta}_{j}\left[h_{j}\left(x_{0}\right)-h_{j}(\tilde{x})\right]+\sum_{k \in K} \frac{\bar{\gamma}_{k}}{w_{k}}\left[\ell_{k}\left(x_{0}\right)-\ell_{k}(\tilde{x})\right] \\
\geq & \sum_{i \in I} \bar{\alpha}_{i}\left\langle\left[x_{i}^{*}+\tilde{v} y_{i}^{*}\right], v\right\rangle+\sum_{j \in J} \bar{\beta}_{j}\left\langle z_{j}^{*}, v\right\rangle+\sum_{k \in K} \bar{\gamma}_{k}\left\langle\xi_{k}^{*}, v\right\rangle .
\end{aligned}
$$

Now using the definition of polar cone, it follows from (7) and $v \in N(\tilde{x}, s)^{\circ}$ that

$$
\sum_{i \in I} \bar{\alpha}_{i}\left\langle\left[x_{i}^{*}+\tilde{v} y_{i}^{*}\right], v\right\rangle+\sum_{j \in J} \bar{\beta}_{j}\left\langle z_{j}^{*}, v\right\rangle+\sum_{k \in K} \bar{\gamma}_{k}\left\langle\xi_{k}^{*}, v\right\rangle \geq 0 .
$$

By (9), (17), (18) and the fact $x_{0} \in \mathbb{F}, \tilde{x} \in \mathbb{F}$, we see that

$$
\sum_{i \in I} \bar{\alpha}_{i}\left[f_{i}\left(x_{0}\right)-\tilde{v} g_{i}\left(x_{0}\right)\right]-\sum_{i \in I} \bar{\alpha}_{i}\left[f_{i}(\tilde{x})-\tilde{v} g_{i}(\tilde{x})\right] \geq 0,
$$

which contradicts (11). This completes the proof.

Now we give an example of minimax fractional programming problem, where to prove optimality the concept of $L$-invexity-infiness may be applied.

Example 3.1. Consider the problem

$$
\min _{x \in \mathbb{F} 1 \leq i \leq 2} \frac{f_{i}(x)}{g_{i}(x)}= \begin{cases}\frac{x^{2}+|x|}{-i+x} ; & \text { if } x \leq 0 \\ \frac{x^{2}+|x|}{-i-x} ; & \text { if } x>0\end{cases}
$$


subject to

$$
h(x)=-|x| \leq 0, \ell(x)=0,
$$

and let $S=R$. Note that the set of feasible solutions of $(\mathrm{P})$ is $\mathbb{F}=R$ and for $\tilde{x}=0 \in \mathbb{F}$, we have $N(\tilde{x}, S)=\{0\}$ and $N(\tilde{x}, S)^{\circ}=R$. It is easy to see that there exist $\tilde{v} \in R_{+}, \bar{\alpha}=$ $\left(\bar{\alpha}_{1}, \bar{\alpha}_{2}\right) \in R_{+}^{2} \backslash\{0\}, \bar{\beta} \in R_{+}$, and $\bar{\gamma} \in R_{+}$such that the relations (4)-(6) hold. Also, for any $x \in S, x_{i}^{*} \in \partial f_{i}(\tilde{x})=\{1,-1\}, y_{i}^{*} \in \partial\left(-g_{i}\right)(\tilde{x})=\{1,-1\}, i=1,2 ; z^{*} \in \partial h(\tilde{x})=\{1,-1\}$ and $\xi^{*} \in \partial \ell(\tilde{x}) \cup \partial(-\ell)(\tilde{x})=\{0\}$, by taking $v=\frac{h(x)-h(\tilde{x})}{z^{*}} \in N(\tilde{x}, S)^{\circ}$, it is not difficult to prove that $(f,-g, h ; \ell)$ is L-invex-infine on $S$ at $\tilde{x}=0$. However, $(f,-g, h ; \ell)$ is not invex-infine [21] on $S$ at $\tilde{x}=0$ (see Chuong [7], Example 3.3). Since all hypotheses of Theorem 3.2 are satisfied, then $\tilde{x}=0$ is optimal in the considered minimax fractional programming problem.

\section{DUALITY}

In this section, we study the following parametric duality model for $(\mathrm{P})$ :

(D) $\max v$

subject to

$$
\begin{gathered}
0 \in \sum_{i \in I} \alpha_{i}\left[\partial f_{i}(y)-v \partial g_{i}(y)\right]+\sum_{j \in J} \beta_{j} \partial h_{j}(y)+\sum_{k \in K} \gamma_{k}\left(\partial \ell_{k}(y) \cup \partial\left(-\ell_{k}\right)(y)\right)+N(y, S), \\
\alpha_{i}\left[f_{i}(y)-v g_{i}(y)\right] \geq 0, \quad i \in I, \\
\beta_{j} h_{j}(y) \geq 0, \quad j \in J, \\
\gamma_{k} \ell_{k}(y) \geq 0, \quad k \in K, \\
y \in S, \quad \alpha \in \Lambda, \quad v \in R_{+}, \quad \beta \in R_{+}^{q}, \quad \gamma \in R_{+}^{r}, \quad \ell(y) \in(\gamma-\Omega(0,\|\gamma\|))^{\circ},
\end{gathered}
$$

where $\Omega(0,\|\gamma\|)=\left\{\sigma \in R^{r}:\|\sigma\|=\|\gamma\|\right\}$. We denote by $\mathbb{W}$ the set of all feasible solutions $(y, \alpha, \beta, \gamma, v) \in S \times \Lambda \times R_{+}^{q} \times R_{+}^{r} \times R_{+}$of problem (D).

The following theorems show that (D) is a dual problem for $(\mathrm{P})$.

Theorem 4.1 (Weak Duality). Let $x \in \mathbb{F}$ and $(y, \alpha, \beta, \gamma, v) \in \mathbb{W}$. Assume also that $(f,-g$, $h ; \ell)$ is L-invex-infine on $S$ at $y$, then $\phi(x) \geq v$.

Proof. Since $(y, \alpha, \beta, \gamma, v) \in \mathbb{W}$ satisfy the relations (19)-(23), there exist $x_{i}^{*} \in \partial f_{i}(\bar{x}), y_{i}^{*} \in$ $\partial\left(-g_{i}\right)(\bar{x}), i \in I, z_{j}^{*} \in \partial h_{j}(\bar{x}), j \in J$ and $\xi_{k}^{*} \in \partial \ell_{k}(\bar{x}) \cup \partial\left(-\ell_{k}\right)(\bar{x}), k \in K$ such that

$$
\begin{gathered}
-\left(\sum_{i \in I} \alpha_{i}\left[x_{i}^{*}+v y_{i}^{*}\right]+\sum_{j \in J} \beta_{j} z_{j}^{*}+\sum_{k \in K} \gamma_{k} \xi_{k}^{*}\right) \in N(y, S), \\
\alpha_{i}\left[f_{i}(y)-v g_{i}(y)\right] \geq 0, \quad i \in I
\end{gathered}
$$




$$
\begin{gathered}
\beta_{j} h_{j}(y) \geq 0, \quad j \in J, \\
\gamma_{k} \ell_{k}(y) \geq 0, \quad k \in K, \\
\langle\gamma-\sigma, \ell(y)\rangle \leq 0, \quad \forall \sigma \in R^{r} \text { with }\|\sigma\|=\|\gamma\| .
\end{gathered}
$$

Suppose to the contrary that

$$
\phi(x)<v .
$$

Using this inequality along with Lemma 2.2, as in the proof of Theorem 3.2, we get

$$
\sum_{i \in I} \alpha_{i}\left(f_{i}(x)-v g_{i}(x)\right)<0 .
$$

By assumption, $(f,-g, h ; \ell)$ is $L$-invex-infine on $S$ at $y$. Then, by Definition 2.8 , there exists $v \in N(y, s)^{\circ}$ such that the following inequalities

$$
\begin{gathered}
f_{i}(x)-f_{i}(y) \geq\left\langle x_{i}^{*}, v\right\rangle, i \in I, \\
-g_{i}(x)+g_{i}(y) \geq\left\langle y_{i}^{*}, v\right\rangle, i \in I, \\
h_{j}(x)-h_{j}(y) \geq\left\langle z_{j}^{*}, v\right\rangle, j \in J, \\
\ell_{k}(x)-\ell_{k}(y)=w_{k}\left\langle\xi_{k}^{*}, v\right\rangle, k \in K,
\end{gathered}
$$

hold for any $x \in \mathbb{F}, x_{i}^{*} \in \partial f_{i}(y), y_{i}^{*} \in \partial\left(-g_{i}\right)(y), i \in I, z_{j}^{*} \in \partial h_{j}(y), j \in J$ and $\xi_{k}^{*} \in$ $\partial \ell_{k}(y) \cup \partial\left(-\ell_{k}\right)(y), k \in K$.

As seen in the proof of Theorem 3.2, the above relations (30)-(33) leads to the following inequality

$$
\begin{aligned}
& \sum_{i \in I} \alpha_{i}\left[f_{i}(x)-v g_{i}(x)\right]-\sum_{i \in I} \alpha_{i}\left[f_{i}(y)-v g_{i}(y)\right] \\
& +\sum_{j \in J} \beta_{j}\left[h_{j}(x)-h_{j}(y)\right]+\sum_{k \in K} \frac{\gamma_{k}}{w_{k}}\left[\ell_{k}(x)-\ell_{k}(y)\right] \geq 0 .
\end{aligned}
$$

Thus, by setting $\sigma_{k}=\frac{\gamma_{k}}{w_{k}}, k \in K$, we have

$$
\begin{aligned}
& \sum_{i \in I} \alpha_{i}\left[f_{i}(x)-v g_{i}(x)\right]-\sum_{i \in I} \alpha_{i}\left[f_{i}(y)-v g_{i}(y)\right] \\
& +\sum_{j \in J} \beta_{j}\left[h_{j}(x)-h_{j}(y)\right]+\sum_{k \in K} \sigma_{k}\left[\ell_{k}(x)-\ell_{k}(y)\right] \geq 0 .
\end{aligned}
$$

From (25), (26) and the fact that $x \in \mathbb{F}$, above inequality yields

$$
\sum_{i \in I} \alpha_{i}\left[f_{i}(x)-v g_{i}(x)\right]-\sum_{k \in K} \sigma_{k} \ell_{k}(y) \geq 0
$$


equivalently,

$$
\sum_{i \in I} \alpha_{i}\left[f_{i}(x)-v g_{i}(x)\right]-\sum_{k \in K} \gamma_{k} \ell_{k}(y)+\langle\gamma-\sigma, \ell(y)\rangle \geq 0,
$$

where $\sigma=\left(\sigma_{1}, \sigma_{2}, \ldots, \sigma_{r}\right) \in R^{r}$. Notice that $\|\sigma\|=\|\gamma\|$ and thus, by (27), (28) and (34), we get

$$
\sum_{i \in I} \alpha_{i}\left[f_{i}(x)-v g_{i}(x)\right] \geq 0
$$

which contradicts (29). This completes the proof.

Theorem 4.2 (Strong Duality). If $\tilde{x}$ is a local optimal solution of $(\mathrm{P})$, and the constraint qualification (CQ) is satisfied at $\tilde{x}$, then there exist $(\tilde{\alpha}, \tilde{\beta}, \tilde{\gamma}, \tilde{v}) \in \Lambda \times R_{+}^{q} \times R_{+}^{r} \times R_{+}$such that $(\tilde{x}, \tilde{\alpha}, \tilde{\beta}, \tilde{\gamma}, \tilde{v})$ is a feasible solution of (D) and the two objectives have the same values. Assume also that the conditions of Theorem 4.1 hold for all feasible solutions of (D), then $(\tilde{x}, \tilde{\alpha}, \tilde{\beta}, \tilde{\gamma}, \tilde{v})$ is a global optimal solution of (D).

Proof. By assumption, $\tilde{x}$ is a local optimal solution of problem (P), and the constraint qualification (CQ) is satisfied at $\tilde{x}$. Then, there exist $\bar{\alpha} \in R_{+}^{p} \backslash\{0\}, \bar{\beta} \in R_{+}^{q}, \bar{\gamma} \in R_{+}^{r}$ and $\tilde{v} \in R_{+}$such that the Karush-Kuhn-Tucker type necessary conditions (Theorem 3.1) are fulfilled at $\tilde{x}$. Thus, we have

$$
\begin{gathered}
0 \in \sum_{i \in I} \bar{\alpha}_{i}\left[\partial f_{i}(\tilde{x})-\tilde{v} \partial g_{i}(\tilde{x})\right]+\sum_{j \in J} \bar{\beta}_{j} \partial h_{j}(\tilde{x})+\sum_{k \in K} \bar{\gamma}_{k}\left(\partial \ell_{k}(\tilde{x}) \cup \partial\left(-\ell_{k}\right)(\tilde{x})\right)+N(\tilde{x}, S), \\
\bar{\alpha}_{i}\left(f_{i}(\tilde{x})-\tilde{v} g_{i}(\tilde{x})\right)=0, i \in I, \\
\bar{\beta}_{j} h_{j}(\tilde{x})=0, j \in J
\end{gathered}
$$

Take

$$
\tilde{\alpha}_{i}=\frac{\bar{\alpha}_{i}}{\sum_{i \in I} \bar{\alpha}_{i}}, i \in I, \quad \tilde{\beta}_{j}=\frac{\bar{\beta}_{j}}{\sum_{i \in I} \bar{\alpha}_{i}}, j \in J, \quad \text { and } \quad \tilde{\gamma}_{k}=\frac{\bar{\gamma}_{k}}{\sum_{i \in I} \bar{\alpha}_{i}}, k \in K
$$

It is easy to see that $\tilde{\alpha}=\left(\tilde{\alpha}_{1}, \tilde{\alpha}_{2}, \ldots, \tilde{\alpha}_{r}\right) \in \Lambda, \tilde{\beta}=\left(\tilde{\beta}_{1}, \tilde{\beta}_{2}, \ldots, \tilde{\beta}_{q}\right) \in R_{+}^{q}$ and $\tilde{\gamma}=$ $\left(\tilde{\gamma}_{1}, \tilde{\gamma}_{2}, \ldots, \tilde{\gamma}_{r}\right) \in R_{+}^{r}$.

Observe that the conditions (35)-(37) are also valid when $\bar{\alpha}_{i}$ 's, $\bar{\beta}_{j}$ 's, and $\bar{\gamma}_{k}$ 's are replaced by $\tilde{\alpha}_{i}$ 's, $\tilde{\beta}_{j}$ 's, and $\tilde{\gamma}_{k}$ 's, respectively.

Since, $\ell_{k}(\tilde{x})=0, k \in K$ for $\tilde{x} \in \mathbb{F}$. Consequently this gives that $\langle\tilde{\gamma}-\sigma, \ell(\tilde{x})\rangle=0$, for all $\sigma \in R^{r}$ with $\|\sigma\|=\|\tilde{\gamma}\|$. That is $\ell(\tilde{x}) \in(\tilde{\gamma}-\Omega(0,\|\tilde{\gamma}\|))^{\circ}$. Therefore, $(\tilde{x}, \tilde{\alpha}, \tilde{\beta}, \tilde{\gamma}, \tilde{v})$ is a feasible solution of (D), moreover, the corresponding objective values of (P) and (D) are equal. The global optimality of $(\tilde{x}, \tilde{\alpha}, \tilde{\beta}, \tilde{\gamma}, \tilde{v})$ for (D) follows from weak duality Theorem 4.1 .

Theorem 4.3 (Strict Converse Duality). Let $\tilde{x}$ and $(\tilde{y}, \tilde{\alpha}, \tilde{\beta}, \tilde{\gamma}, \tilde{v})$ be optimal solutions of (P) and (D), respectively, and assume that the assumptions of Theorem 4.2 are fulfilled. Also, assume that $(f,-g, h ; \ell)$ is L-strictly invex-infine on $S$ at $\tilde{y}$, then $\tilde{x}=\tilde{y}$; that is, $\tilde{y}$ is an optimal solution of $(\mathrm{P})$. 
Proof. Suppose to the contrary that $\tilde{x} \neq \tilde{y}$. By Theorem 4.2, it follows that

$$
\phi(\tilde{x})=\tilde{v} .
$$

Now, proceeding as in Theorem 4.1, we see that the $L$-strictly invex-infine of $(f,-g, h ; \ell)$ on $S$ at $\tilde{y}$, yields the following inequality

$$
\sum_{i \in I} \tilde{\alpha}_{i}\left[f_{i}(\tilde{x})-\tilde{v} g_{i}(\tilde{x})\right]>0
$$

Using this inequality along with Lemma 2.2, we see that

$$
\phi(\tilde{x})=\max _{\hat{\alpha} \in \Lambda} \frac{\sum_{i \in I} \hat{\alpha}_{i} f_{i}(\tilde{x})}{\sum_{i \in I} \hat{\alpha}_{i} g_{i}(\tilde{x})} \geq \frac{\sum_{i \in I} \tilde{\alpha}_{i} f_{i}(\tilde{x})}{\sum_{i \in I} \tilde{\alpha}_{i} g_{i}(\tilde{x})}>\tilde{v},
$$

which contradicts (38). This completes the proof.

\section{CONCLUSION}

In this paper, we have established optimality conditions and duality results for a class of nonsmooth minimax fractional programming problems possessing $L$-invex-infiness property. This paper extends entirely earlier works, in which optimality conditions and duality results have been obtained in terms of the Clarke generalized gradient for a generalized optimization problems (for example, the results of Ahmad [2], Antczak [3], Zalmai [26] and Zheng and Cheng [27]). We are going to extend the results established in the paper to a larger class of nonsmooth variational and nonsmooth control problems. This will orient the future research of the author.

\section{ACKNOWLEDGMENTS}

The authors are grateful to the referees for their valuable suggestions that helped to improve this article in its present form. The research of the first author is financially supported by the DST, New Delhi, India under (F. No. SR/FTP/MS-007/2011).

\section{REFERENCES}

[1] Ahmad I \& Husain Z. 2006. Optimality conditions and duality in nondifferentiable minimax fractional programming with generalized convexity, J. Optim. Theory Appl., 129: 255-275.

[2] Ahmad I. 2011. Nonsmooth minimax programming with $(\Phi, \rho)$-invex functions, In Proceeding of the Annual International Conference on Operations Research and Statistics, Penang, Malaysia, $168-173$.

[3] AntcZaK T. 2011. Nonsmooth minimax programming under locally Lipschitz $(\Phi, \rho)$-invexity, Appl. Math. Comput., 217: 9606-9624.

[4] AntcZaK T \& StAsiak A. 2011. ( $\Phi, \rho)$-invexity in nonsmooth optimization, Numer. Funct. Anal. Optim., 32: 1-25. 
[5] BRito JAM \& XAVIER AE. 2006. Modelagens min-max-min para o problema de localização de estações de rádio base, Pesqui. Oper., 26(2): 295-319.

[6] Caristi G, Ferrara M \& Stefanescu A. 2006. Mathematical programming with $(\Phi, \rho)$ invexity. In: Generalized Convexity and Related Topics, Lecture Notes in Economics and Mathematical Systems, Vol. 583. (Konnov IV, LuC DT \& Rubinov AM, eds.), Springer, Berlin-HeidelbergNew York, 167-176.

[7] Chuong TD. 2012. L-invex-infine functions and applications, Nonlinear Anal. Theory Methods Appl., 75: 5044-5052.

[8] Chuong TD \& KIM DS. Nondifferentiable minimax programming problems with applications, Ann. Oper. Res., DOI:10.1007/s10479-015-1843-3.

[9] Danskin JM. 1967. The Theory of Max-Min and its Applications to Weapon Allocation Problems, Springer-Verlag, New York, NY, USA.

[10] Demyanov VF \& Molozemov VN. 1974. Introduction to Minmax, John Wiley and Sons, New York.

[11] Dhara A \& Mehra A. 2010. Approximate optimality conditions for minimax programming problems, Optimization, 59: 1013-1032.

[12] Du D, Pardalos PM \& Wu WZ. 1995. Minimax and Applications, Kluwer Academic, Dordrecht.

[13] Gupta SK, DAngar D \& AhMAD I. 2014. On second order duality for nondifferentiable minimax fractional programming problems involving type-I functions, ANZIAM J., 55(EMAC2013): C479C494.

[14] Hanson MA. 1981. On sufficiency of the Kuhn-Tucker conditions, J. Math. Anal. Appl., 80: $545-550$.

[15] Ho SC \& LAI HC. 2014. Mixed-type duality on nonsmooth minimax fractional programming involving exponential $(p, r)$-invexity, Numer. Funct. Anal. Optim., 35: 1560-1578.

[16] LIU X \& YUAN D. 2014. Minimax fractional programming with nondifferentiable $(G, \beta)$-invexity, Filomat, 28(10): 2027-2035.

[17] LIU Q \& WANG J. 2015. A projection neural network for constrained quadratic minimax optimization, IEEE Trans. Neural Netw. Learn. Syst., 26: 2891-2900.

[18] Mordukhovich BS. 2006. Variational analysis and generalized differentiation, I: basic theory, Berlin, Springer.

[19] Mordukhovich BS \& Mou L. 2009. Necessary conditions for nonsmooth optimization problems with operator constraints in metric spaces, J. Convex Anal., 16: 913-937.

[20] Nobakhtian S. 2006. Infine functions and nonsmooth multiobjective optimization problems, Comput. Math. Appl., 51: 1385-1394.

[21] SACH PH, LEE GM \& Kim DS. 2003. Infine functions, nonsmooth alternative theorems and vector optimization problems, J. Global Optim., 27: 51-81.

[22] Soleimani-Damaneh M. 2010. Nonsmooth optimization using Mordukhovich's subdifferential, SIAM J. Control Optim., 48: 3403-3432.

[23] KAILEY SN \& SHARMA V. On second order duality of minimax fractional programming with square root term involving generalized $B$ - $(p, r)$-invex functions, DOI: 10.1007/s10479-016-2147-y. 
[24] Stancu-Minasian IM. 2002. Optimality and duality in fractional programming involving semilocally preinvex and related functions, J. Inform. Optim. Sci., 23: 185-201.

[25] Rockafellar RT. 1970. Convex Analysis, Princeton, NJ, Princeton University Press.

[26] ZALMAI GJ. 1995. Optimality conditions and duality models for generalized fractional programming problems containing locally subdifferentiable and $\rho$-convex functions, Optimization, 32: 95-124.

[27] Zheng XJ \& Cheng L. 2007. Minimax fractional programming under nonsmooth generalized ( $\mathscr{F}, \rho, \theta)$-d-univexity, J. Math. Anal. Appl., 328: 676-689. 